\title{
Integrative analysis of differential miRNA and functional study of miR-21 by seed-targeting inhibition in multiple myeloma cells in response to berberine
}

\author{
Xiaochuang Luo ${ }^{1+}$, Jingyi Gu${ }^{1 \dagger}$, Rongxuan Zhu ${ }^{2 \dagger}$, Maoxiao Feng ${ }^{1}$, Xuejiao Zhu', Yumin $\mathrm{Li}^{1}$ and Jia Fei ${ }^{1 *}$
}

\begin{abstract}
Background: Berberine is a natural alkaloid derived from a traditional Chinese herbal medicine. It is known to modulate microRNA (miRNA) levels, although the mechanism for this action is unknown. Here, we previously demonstrate that the expression of 87 miRNAs is differentially affected by berberine in multiple myeloma cells. Among 49 miRNAs that are down-regulated, nine act as oncomirs, including miR-21. Integrative analysis showed that 28 of the down-regulated miRNAs participate in tumor protein p53 (TP53) signaling and other cancer pathways. miR-21 is involved in all these pathways, and is one of the most important oncomirs to be affected by berberine in multiple myeloma cells.

Results: We confirmed that berberine down-regulated miRNA-21 expression and significantly up-regulated the expression of programmed cell death 4 (PDCD4), a predicted miR-21 target. Luciferase reporter assays confirmed that PDCD4 was directly regulated by miR-21. Bioinformatic analysis revealed that the miR-21 promoter can be targeted by signal transducer and activator of transcription 3 (STAT3). Down-regulation of interleukin 6 (IL6) by berberine might lead to inhibition of miR-21 transcription through STAT3 down-regulation in multiple myeloma. Furthermore, both berberine and seed-targeting anti-miR-21 oligonucleotide induced apoptosis, G2-phase cell cycle arrest and colony inhibition in multiple myeloma cell lines. Depletion of PDCD4 by short interfering RNA could rescue berberine-induced cytotoxicity in multiple myeloma cells.
\end{abstract}

Conclusions: Our results suggest that berberine suppresses multiple myeloma cell growth, at least in part, by down-regulating miR-21 levels possibly through IL6/STAT3. This led to increased PDCD4 expression, which is likely to result in suppression of the p53 signaling pathway. These findings may also provide new mechanistic insight into the anti-cancer effects of certain compounds in traditional Chinese herbal medicines.

Keywords: Berberine, miRNA-21, Multiple myeloma, Programmed cell death 4, Bioinformatic, Tumor protein p53, Cell cycle, Apoptosis

\section{Background}

Multiple myeloma (MM) is a clonal B cell malignancy characterized by proliferation of plasma cells (PCs) within the bone marrow (BM). Globally, its incidence varies from 1 per 100,000 people in China to about 4 per 100,000 people in most developed countries [1,2]. MM is characterized by profound genomic instability involving both

\footnotetext{
* Correspondence: efeijia@163.com

${ }^{\dagger}$ Equal contributors

'Department of Biochemistry and Molecular Biology, Medical College of Jinan University, Guangzhou 510632, China

Full list of author information is available at the end of the article
}

numerical and structural chromosomal aberrations of potential prognostic relevance [1,2]. Nearly half of MM tumors are hyperdiploid (HD) with multiple trisomies of non-random odd-numbered chromosomes, a low prevalence of chromosomal translocations involving the immunoglobulin heavy chain (IgH) locus at $14 \mathrm{q} 32$ and chromosome 13 deletion [3]. It has been suggested that chromosomal abnormalities and other types of genetic or epigenetic alterations might contribute to miRNA deregulation in cancer [4-6]. 
miRNAs are a newly discovered class of endogenous non-coding small RNAs that regulate gene expression through degrading target mRNAs and/or suppressing their translation by binding to the 3 '-untranslated region (3'UTR) of target genes. Bioinformatic predictions indicate that $30 \%$ of all human genes are regulated by miRNAs. Thus, miRNAs are involved in a variety of biological processes, from development and differentiation to survival, apoptosis, and senescence [7-9].

Accumulating evidence suggests that miRNAs that are significantly over-expressed in tumors may be a novel class of oncogene. Termed "oncomirs", these oncogene miRNAs usually promote tumor development by negatively regulating tumor suppressor genes that control various biological processes. Therefore, altering oncomir expression might be a valuable strategy for cancer treatment $[10,11]$.

Differential miRNA expression and high levels of oncomirs, including miR-21, miR-155, miR-17-92, and miR125b, have been reported in MM. miR-21 is frequently over-expressed in $\mathrm{MM}$ and is involved in proliferation, apoptosis, cell cycle, drug-resistance, and pathogenesis [6,12-15]. Loffler et al. demonstrated that interleukin-6 (IL6) regulates miR-21 transcription in IL6-dependent human myeloma cell lines (HMCLs) through a signal transducer and activator of transcription 3 (STAT3)-related mechanism. Importantly, ectopic expression of miR-21 was sufficient to sustain growth of IL6-dependent MM cells in the absence of IL6 [16]. This evidence indicates that miR-21 is an important oncomir in MM.

Berberine (BB), an alkaloid that was initially isolated from Chinese herbs, is currently used as a traditional medicine to treat diarrhea caused by bacteria, although the mechanism for this action is unknown. Accumulating evidence suggests that BB also elicits anti-cancer effects by inhibiting cell growth and inducing apoptosis in a variety of cancer cell lines [17-20]. Animal studies have shown that BB can inhibit chemical-induced carcinogenesis, tumor promotion, and tumor invasion [21,22]. Recent studies also show that $\mathrm{BB}$ exerts anti-cancer effects by inhibiting proliferation and reproduction of certain tumorigenic microorganisms and viruses, such as Helicobacter pylori and hepatitis B. BB can also regulate the transcription of some oncogenes and carcinogenesis-related genes via interactions with DNA and RNA. Furthermore, BB is a broad-spectrum enzyme inhibitor that affects $N$-acetyltransferase, cyclooxygenase-2, and topoisomerase activities, as well as gene expression and protein synthesis [23]. Thus, BB can regulate many oncogenic mRNAs and proteins. However, whether BB can regulate miRNAs remains unknown.

miRNAs in animals have a highly conserved 5 '-end sequence consisting of 7-8 nt called the seed sequence. The seed sequence binds with $100 \%$ complementarity to the target mRNA and is a key feature in the recognition between a miRNA and its target mRNA [24]. Inhibition of the seed sequence leads to a loss of mature miRNA function, and is the target of anti-miRNA oligonucleotides (AMOs) [25,26].

In this research, we performed microarray analysis to explore the possibility that $\mathrm{BB}$ regulates miRNA expression. Our results show that $\mathrm{BB}$ differentially regulates the expression of a number of miRNAs. Forty-nine miRNAs were down-regulated, of which 28 were shown by KEGG analysis to be involved in p53 signaling, the cell cycle and other cancer pathways. Of the 49 miRNAs, miR-21 had the most target genes and participates in all the signaling pathways and can, therefore, be considered as one of the most important oncomirs. The role of miR-21 in MM was further investigated with the use of AMO-miR-21. Our findings provide new insight into anti-cancer mechanisms of traditional Chinese herbal medicines and provide evidence that they are effective in treating cancer.

\section{Methods}

\section{Microarray analysis of miRNA expression}

Based on our preliminary study, the MM cell line, RPMI8266 , was treated with $75 \mu \mathrm{M}$ BB for $48 \mathrm{~h}$. Total miRNA from $1 \times 10^{8}$ cells was isolated and labeled using an mirVANA $^{\mathrm{Tm}}$ miRNA Isolation kit and mirVANA ${ }^{\mathrm{mm}}$ miRNA labeling kit (Ambion, Austin, TX, USA).. Samples $(4 \mu \mathrm{g})$ labeled with $\mathrm{Cy} 3 / \mathrm{Cy} 5$ were hybridized on miRNA microarrays (CSC-GE-3, chipscreen biosciences, Shenzhen, China). After air drying, each chip was scanned with a Generation III array scanner (Amersham Pharmacia). Data analyses were performed using Imagequant 5.0 (Array Vision 6.0).

\section{Bioinformatic analysis}

miRFocus software (http://mirfocus.org), developed by LC Science USA, was used to analyze miRNA-target gene pathways and to determine related miRNA annotations (Additional file 1: Figure S1).

\section{Oligonucleotides}

An anti-miR-21 oligonucleotide (AMO-miR-21) was designed according to sequence complementary to mature miRNA-21: AMO-miR-21, 5'-ATAAGCTA-3' (8 bp). A control scramble AMO (SCR) 5' -TCATACTA-3' (8 bp) was also synthesized (Additional file 1: Figure S2). All oligodeoxynucleotides were chemically synthesized and modified with phosphorothioate and/or fluorescein isothiocyanate (FITC) by the Shanghai Sangon Bio-engineering Company, China. The siRNA sequence of PDCD4 (siPDCD4) was 5' -AAGGUGGCUGGAACAUCUAUU-3'. The RNA duplexes were synthesized and purified by Shanghai GenePharma Company, China.

\section{Cell lines, transfection and cell culture reagents}

MM cell lines (RPMI-8266 and U226) were obtained from the Shanghai Institute of Cell Biology, China. The 
cells were cultured in RPMI containing 25 mM HEPES, $10 \%$ fetal bovine serum (FBS), $0.05 \mathrm{mM}$ 2-mercaptoethanol, $1 \mathrm{mM}$ sodium pyruvate, $2 \mathrm{mM}$ L-glutamine, $100 \mathrm{U} / \mathrm{mL}$ penicillin, and $50 \mathrm{U} / \mathrm{mL}$ streptomycin. The cells were grown in RPMI-1640 medium containing $10 \%$ fetal calf serum (FCS) at $37^{\circ} \mathrm{C}$ in a $5 \% \mathrm{CO}_{2}$ humidified atmosphere (Thermo FORMA 3110).

BB was purchased from Sigma-Aldrich. RPMI-8266 and U226 cells in the exponential phase of growth were seeded in 96- or 24-well plates (Costar) and transfected with $0.5 \mu \mathrm{M}$ AMO-miR-21 using Lipofectamine 2000 (Invitrogen) in serum-free RPMI-1640. PDCD4 siRNA and control SCR (100 nM) were transfected into RPMI-8266 and U226 cells using Lipofectamine 2000 according to the manufacturer's instructions.

\section{Luciferase reporter assays}

The full-length human PDCD4 3'-UTR (1917 bp) was PCRamplified from cDNA with the following primers: $5^{\prime}-\mathrm{C}$ cgctcgagATATAAGAACTCTTGCAGTCT-3' and 5' -ataaga atgcggccgcACAGAGGATCTTTACATGTTTA-3' containing NotI and XhoI restriction site overhangs, respectively (indicated in italic type). The amplified product, which contains one putative miR-21 binding site, was cloned into psiCHECK-2 (Promega) immediately downstream of the Renilla luciferase reporter gene and was named PDCD4 3'UTR. Site-directed mutagenesis was performed using the QuikChange II XL site-directed mutagenesis kit (Stratagene) to change three nucleotides in the seed sequence, in which ATAAGCTA was substituted by TAGCTACT. The mutant plasmid was named PDCD4-mut- 3'-UTR.

RPMI-8266 cells were cotransfected with $100 \mathrm{nM}$ miR-21 or $0.5 \mu \mathrm{M}$ AMO-miR-21 together with PDCD4 3'-UTR or PDCD4-mut-UTR and assayed for luciferase activity $24 \mathrm{~h}$ post-transfection using the Dual-Luciferase Reporter Assay System (E1910, Promega). For each sample, firefly luciferase activity was normalized against Renilla luciferase activity.

\section{Real-time PCR assay}

RPMI-8266 and U226 cells were treated with $75 \mu \mathrm{M}$ and $120 \mu \mathrm{M} \mathrm{BB}$, respectively. Total RNA was extracted in TRIzol (Invitrogen). The levels of miR-21 and U6 small nuclear RNA (snRNA) were determined using a miRNA RT-PCR Quantitation Kit (Shang Hai Gene Pharma Company). U6 snRNA was used as the internal control, and the fold-change in miR-21 expression was calculated using the $2^{-\Delta \Delta C T}$ method.

RPMI-8266 cells were transfected with $0.5 \mu \mathrm{M}$ AMOmiR-21 using Lipofectamine 2000 and cultured for $48 \mathrm{~h}$. Levels of PDCD4 mRNA were determined using SYBRGreen real-time PCR assays. PDCD4 primers used were 5' -CCAAAGAAAGGTGGTGCA-3' and 5' -TGAGGTAC TTCCAGTTCC-3' and GAPDH primers were 5' ${ }^{\prime}$-CAA CGGATTTGGTCGTATT-3' and 5'-CACAGTCTTCTGG
GTGGC-3'. PDCD4 mRNA levels were normalized to those of GAPDH.

\section{Western blot analysis and enzyme-linked immunosorbent assay (ELISA)}

Cells were lysed in radioimmunoprecipitation assay (RIPA) buffer in the presence of proteinase inhibitor (Biocolor BioScience \& Technology Company, Shanghai, China). Cell lysates $(30 \mu \mathrm{g})$ were denatured in Laemmli sample buffer (Bio-Rad) for $5 \mathrm{~min}$ at $95.1^{\circ} \mathrm{C}$, separated by $10 \%$ SDS-PAGE and transferred to nitrocellulose membranes. Membranes were blocked with $5 \%(\mathrm{w} / \mathrm{v})$ fat-free milk in phosphatebuffered saline (PBS) and $0.5 \%(\mathrm{v} / \mathrm{v})$ Tween-20 for $1 \mathrm{~h}$, and then incubated with anti-PDCD4 antibody (Cell Signaling Technology) at room temperature for $2 \mathrm{~h}$. After washing, membranes were incubated with horseradish peroxidaseconjugated secondary antibody. Signals were visualized with enhanced chemiluminescence (ECL) (BeyoECL Plus, Beyotime), and analyzed using a BI-2000 system (Beyotime, Haimen, Jiangsu province, China),

RPMI-8266 and U226 cells were treated with $75 \mu \mathrm{M}$ and $120 \mu \mathrm{M} \mathrm{BB}$, respectively.

IL6 protein levels in supernatants were determined using a human IL6 ELISA kit (R\&D Systems).

\section{MTT assay}

RPMI-8266 cell viability was determined by 3-(4,5-dimethylthiazol-2-yl)-2,4- diphenyl-tetrazolium bromide (MTT) assays. Briefly, cells were seeded at a density of $1 \times 10^{5}$ cells/ml in 96-well plates $(100 \mu \mathrm{l} /$ well). The cells were treated with BB $(75 \mu \mathrm{M})$ or AMO-miR-21 $(0.5 \mu \mathrm{M})$. At $48 \mathrm{~h}$ post-treatment, $20 \mu \mathrm{l}$ MTT stock solution $(5 \mathrm{mg} / \mathrm{ml})$ was added to each well, and the plate was incubated for $4 \mathrm{~h}$ at $37^{\circ} \mathrm{C}$. The media was then removed, and dimethyl sulfoxide (DMSO) $(150 \mu \mathrm{l})$ was added to dissolve the blue formazan crystals produced by viable cells. Cell viability was assessed by measuring the absorbance at $570 \mathrm{~nm}$ on a Bio-Rad microtiter plate reader.

\section{Colony assay}

The colony assay for dispersed single cells was performed to measure the capacity of cells to form colonies. Cells treated with $\mathrm{BB}(75 \mu \mathrm{M})$ or AMO-miR-21 $(0.5 \mu \mathrm{M})$ were seeded onto a 24 -well plate $\left(2 \times 10^{3}\right.$ cells per well $)$ and mixed thoroughly with $0.9 \%$ methylcellulose solution in RPMI-1640 containing 20\% FBS. Single cells were randomly and evenly distributed throughout each well. Colonies were formed during incubation for 1-2 weeks at $37^{\circ} \mathrm{C}$ in a $5 \% \mathrm{CO}_{2}$ humidified atmosphere. Light microscopy was used to observe and count colonies containing more than 50 cells. 


\section{Flow cytometry}

Flow cytometry (Coulter Elite; Fullerton, CA, USA) was performed to analyze cell cycle profiles and levels of apoptosis. For cell cycle analysis, cells were collected, rinsed twice with PBS, fixed in $70 \%$ ethanol for $1 \mathrm{~h}$ at $4{ }^{\circ} \mathrm{C}$ and stained with propidium iodide (PI) solution $(50 \mu \mathrm{g} / \mathrm{ml})$ containing RNAse A (200 ug/ml). Cell cycle was analyzed using flow cytometry according to DNA content. To analyze apoptosis, cells were stained with fluorescein isothiocyanate (FITC)-conjugated annexin V and PI. For each sample, data from approximately 10,000 cells were recorded in the list mode on logarithmic scales. Apoptotic and necrotic cells were analyzed by performing quadrant statistics on PI-negative/annexin V-positive cells and PI/ annexin $\mathrm{V}$ double-positive cells, respectively.

\section{Results}

BB modulates miRNA expression in MM cells

Of the 1152 miRNAs represented on the microarray, 87 were differentially expressed between BB-treated and control cells (Additional file 1: Figure S3); 49 were downregulated, and 38 were up-regulated compared to control. Further analysis revealed that nine of the 49 downregulated miRNAs were potential oncomirs, and three of the 38 up-regulated miRNAs are considered tumor suppressor genes (Additional file 2: Table S1 and Additional file 2: Table S2). Among the 49 down-regulated miRNAs, mirFocus software identified 28 that are involved in p53 signaling, the cell cycle and other cancer pathways (Figure 1). Of these, miR-21, has the most identified target genes and participates in all the above signaling pathways. Thus mir21 can be considered as one of the most important oncomirs. These results suggest that BB suppression of MM might involve miRNA-mediated gene expression.

Regulation of miRNA-21, IL6 and PDCD4 levels by berberine and the effects of exogenous IL6 on miRNA-21 expression in MM cells

To validate down-regulation of miR-21 by $\mathrm{BB}$ and to investigate the effect of exogenous IL6 on BB-modulated miRNA-21 expression in MM, RPMI-8266 and U226 cells were treated without or with $75 \mu \mathrm{M}$ or $120 \mu \mathrm{M}$ BB, respectively, in the absence or presence of IL6 $(0.5 \mathrm{ng} / \mathrm{ml})$. Total RNA and/or protein was isolated and analyzed for the expression of miR-21 and PDCD4 using real-time PCR and western blot analyses. As shown in Figure 1, BB treatment significantly down-regulated miR-21 levels in a dosedependent manner, and exogenous IL6 somewhat rescued BB-mediated down-regulation of miR-21 (Figure 2A). Interestingly, BB also reduced the level of IL6 in supernatant (Figure 2B). IL6 is an important oncogene in MM and is involved in miR-21 transcription [16]. These results confirmed that BB down-regulates miR-21 and IL6 and consequently the miRNA target gene, $P D C D 4$ was up-regulated (Figure 2C, 2D). Meanwhile, exogenous IL6 could rescue BB-induced miRNA-21 down-regulation in MM cells.

\section{BB down-regulates STAT3 mRNA levels in MM cells}

Bioinformatic analysis showed that sequence upstream of the miR-21 gene contained two putative STAT3 binding sites (Additional file 1: Figure S4A). To investigate whether BB affects STAT3 mRNA levels, RPMI-8266 and U226 cells were treated with $75 \mu \mathrm{M}$ and $120 \mu \mathrm{M}$ $\mathrm{BB}$, respectively. STAT3 mRNA levels were significantly

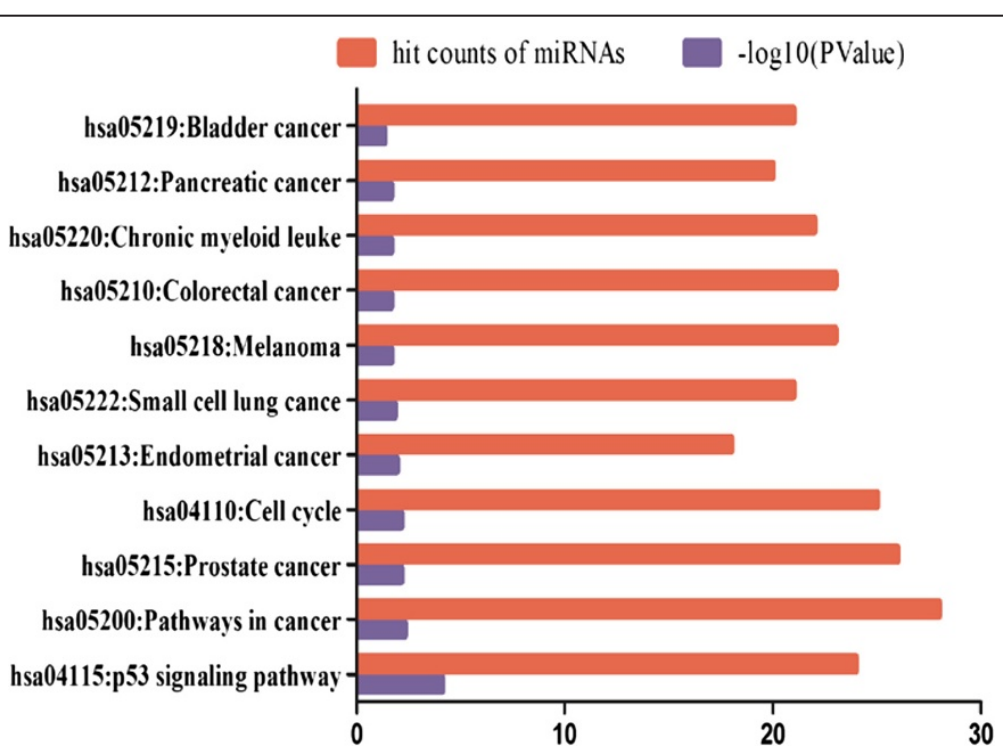

Figure 1 Ingenuity analysis of miRNA predictive pathways. Among the 49 down-regulated miRNAs, 28 were shown by mirFocus to be involved in p53 signaling, cell cycle and other cancer pathways. miR-21 participates in all the above signaling pathways, and can be considered as one of the most important oncomirs in the effects of BB on MM. 


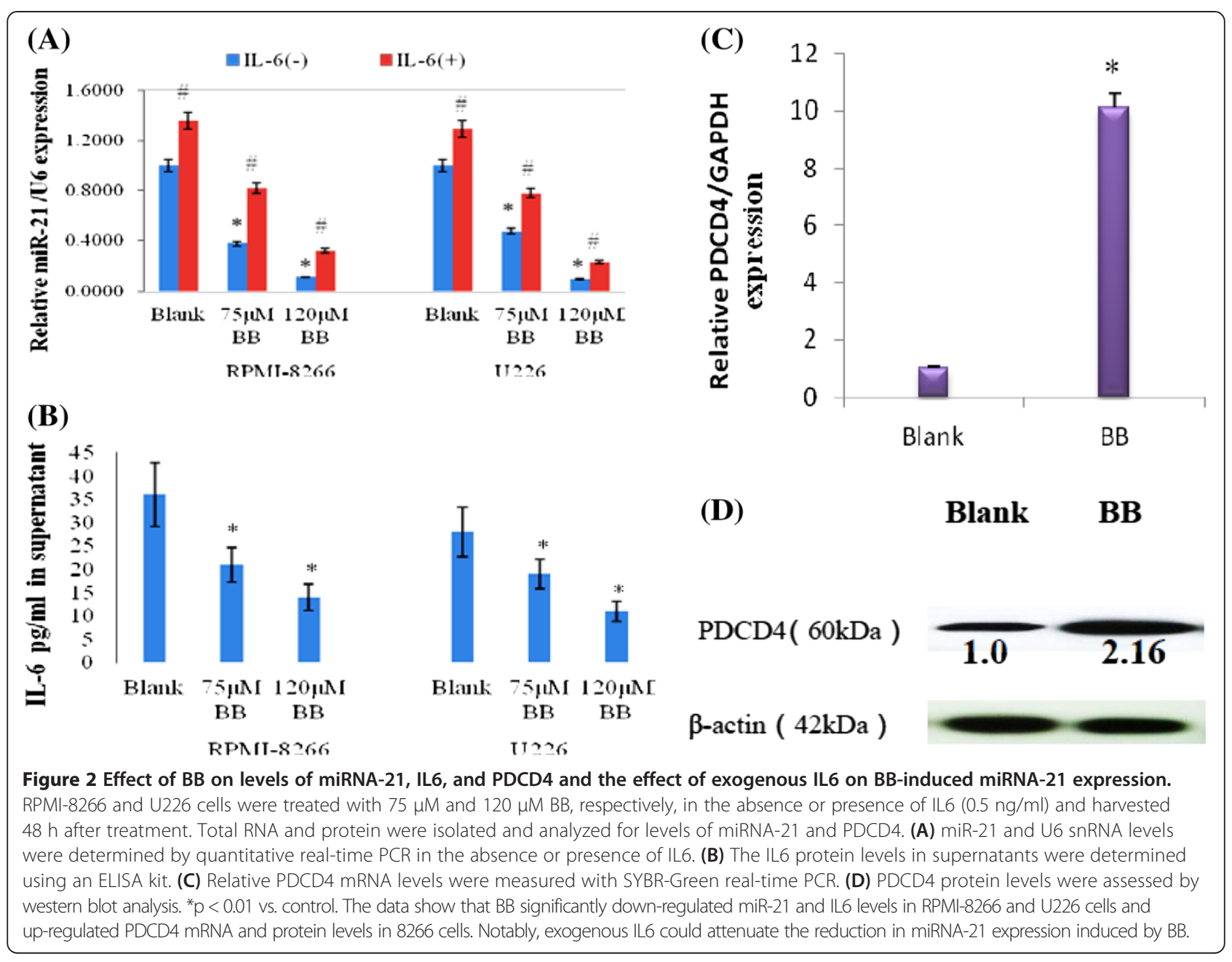

decreased (Additional file 1: Figure S4B). STAT3 is recruited to the miR-21 regulatory region in response to IL6 [16]; therefore, BB-mediated down-regulation of IL6 might cause transcriptional inhibition of miR-21 via reduced STAT3 action.

\section{PDCD4 is a direct target of miRNA-21}

Because BB treatment reduced miRNA-21 levels in MM cells, we next examined whether $\mathrm{BB}$ also regulated the expression of genes targeted by miRNA-21. We first focused on the PDCD4 gene, which is known to be targeted by miR-21. AMO-miR-21 was transfected into RPMI-8266 cells to knock down endogenous mir-21. As shown in Additional file 1: Figure S5A and S5B, PDCD4 mRNA and protein levels were significantly increased in AMO-miR21-transfected cells compared with control-transfected cells, suggesting that $P D C D 4$ is regulated by miR-21.

We performed luciferase reporter assays to determine whether PDCD4 is a direct target of miR-21. The luciferase reporter plasmid containing the $3^{\prime}$-UTR of PDCD4
(PDCD4-3'UTR) or a mutant PDCD4 3'-UTR containing a mutation in the putative miR-21 binding site (PDCD4-mut-3'UTR) were co-transfected with AMOmiR-21 or a miRNA-21 mimic. The firefly luciferase plasmid was used as an internal control. As shown in Additional file 1: Figure S5C, over-expression of the miR-21 mimic significantly suppressed luciferase activity, whereas transfection of AMO-miR-21 significantly increased luciferase activity in cells transfected with PDCD4-3'UTR. In contrast, luciferase activities in cells transfected with PDCD4-mut-3'UTR were not significantly changed by either over-expression or knockdown of miR21 (Additional file 1: Figure S5C,S5D). These results indicate that PDCD4 might be a direct target of miR-21.

\section{Transfection efficiency and localization of AMO-mir-21 in} RPMI-8266

AMO-mir-21 was modified with FITC, and transfected into RPMI-8266 cells using Lipofectamine 2000. High levels of AMO-mir-21-FITC were detected, mainly in the cytoplasm 
(Additional file 1: Figure S6A). Flow cytometry showed that at $24 \mathrm{~h}$ and $48 \mathrm{~h}$ post-transfection $95.27 \%$ and $85.8 \%$ of cells, respectively, were FITC-positive (Additional file 1: Figure S6B).

BB and AMO-miR-21 inhibit cell growth and induce apoptosis To determine the effects of $\mathrm{BB}$ on $\mathrm{MM}$ cell growth via miR-21, RPMI-8266 cells were treated with BB $(75 \mu \mathrm{M})$ or AMO-miR-21 $(0.5 \mu \mathrm{M})$ as described in Materials and Methods. Cell viability was assessed by triplicate MTT assays. As shown in Figure 3A, treatment with BB at $50 \mu \mathrm{M}$ or higher significantly inhibited cell proliferation. Similar results were also observed when miR-21 was knocked down by AMO-miR-21 (Figure 3A), and these effects were dose-dependent. Thus, both BB and AMO-miR-21 inhibited MM cell viability, indicating that the BB-mediated cell death might involve miR-21 down-regulation.

We next investigated whether BB treatment or miR-21 inhibition could induce apoptosis in MM cells. To this end, RPMI-8266 cells were treated with BB or transfected with AMO-miR-21. The cells were then stained with annexin V and PI and analyzed by flow cytometry. As

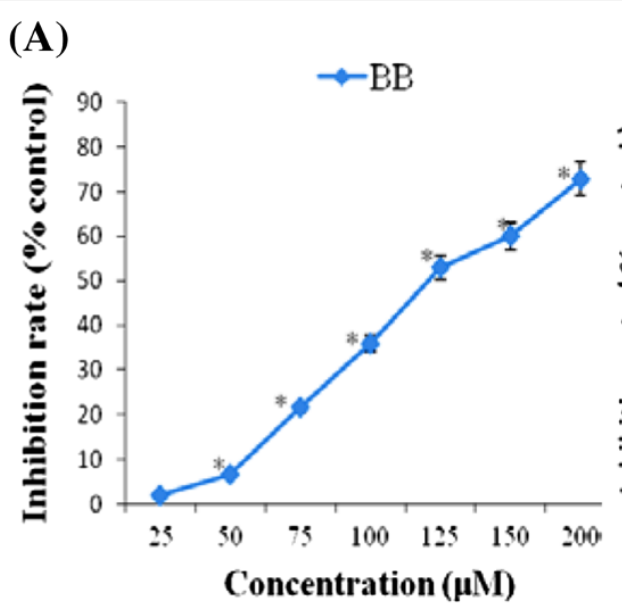

(B)
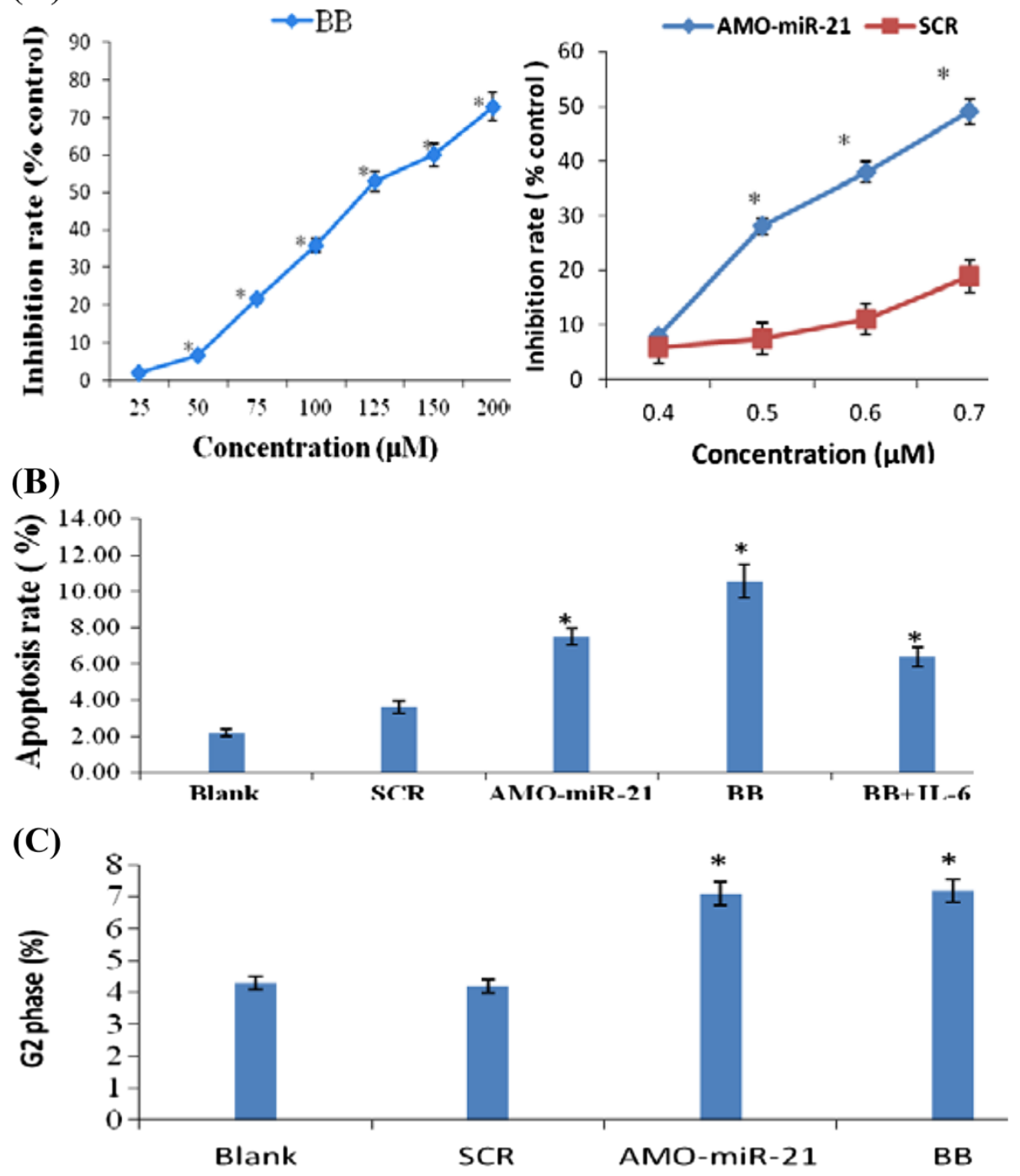

Figure 3 BB and AMO-miR-21 inhibit cell viability and induce apoptosis and G2 phase cell cycle arrest. RPMI-8266 cells were treated with various concentrations of BB and AMO-miR-21 then plated in 96-well plates in medium containing 10\% FCS and cultured for another $48 \mathrm{~h}$. Cell viability was assessed by triplicate MTT assays. (A) BB and AMO-miR-21. ${ }^{*} p<0.01$ vs. blank and Scramble controls. Both BB and AMO-miR-21 inhibited cell viability in a dose-dependent manner. RPMI-8266 cells were treated with $75 \mu \mathrm{M}$ BB or $0.5 \mu \mathrm{M}$ AMO-miR-21. Cells were then stained with FITC-conjugated annexin $\checkmark$ and PI for 15 min., and then analyzed by flow cytometry. (B) Both BB and AMO-miR-21 promoted apoptosis and IL6 could reduce BB-induced apoptosis. ${ }^{*} p<0.01$ vs. control. (C) Both BB and AMO-miR-21 significantly induced G2-phase cell cycle arrest. ${ }^{*} p<0.01$ vs. blank or SCR controls. 
shown in Figure 3B and Additional file 1: Figure S7, both treatments significantly increased the number of apoptotic cells, indicating that $\mathrm{BB}$ treatment and miR-21 inhibition induce apoptosis.

\section{BB and AMO-miR-21 induce G2-phase cell cycle arrest}

To investigate the effect of $\mathrm{BB}$ treatment or miR-21 inhibition on the cell cycle, RPMI-8266 cells were treated with $\mathrm{BB}$ or transfected with AMO-miR-21 and then subjected to cell cycle analysis by flow cytometry. As shown in Figure 3C and Additional file 1: Figure S8, BB treatment resulted in an accumulation of cells in the G2/M phase. Interestingly, miR-21 inhibition by AMO-miR-21 produced almost identical effects on the cell cycle to those observed following $\mathrm{BB}$ treatment. These results indicate that BB-induced G2/M arrest in MM cells is at least partially mediated via miR-21 down-regulation.

\section{$B B$ and AMO-miR-21 suppress colony formation}

Colony growth is closely related to neoplastic capacity. To investigate the effect of $\mathrm{BB}$ and $\mathrm{AMO}-\mathrm{miR}-21$ on colony formation in RPMI-8266 cells, colony growth assays were performed. One week post-treatment, we found that RPMI-8266 cells treated with AMO-miR-21 or BB showed fewer colonies, compared to the control groups (Figure 4). These results indicate that both BB and miR-21 knockdown had an inhibitory effect on the malignant growth capacity of MM cells.

\section{Testing siRNAs for their ability to silence PDCD4}

To examine whether miR-21 down-regulation is associated with BB-induced cytotoxicity, we further studied the effect of PDCD4 knockdown in MM cells. We designed three siRNAs against the PDCD4 gene and evaluated their knockdown efficiency using real-time PCR analysis. We found that one siRNA efficiently knocked down PDCD4 mRNA and down-regulated PDCD4 protein levels in RPMI-8266 cells (Figure 5A,B).

\section{PDCD4 siRNA ameliorates berberine-induced inhibition of MM cell growth}

Next, we examined whether PDCD4 knockdown could rescue the cytotoxic effect of BB treatment. RPMI-8266 and U226 cells transfected with $P D C D 4$ or control siRNA were treated with $B B$ for $72 \mathrm{~h}$, and cell viability was assessed by MTT assays. As shown in Figure 5C,D, PDCD4 siRNA transfection alone did not affect cell viability. However, PDCD4 depletion significantly ameliorated BB-induced growth inhibition, indicating that PDCD4 could be a downstream effector protein in the BB-induced cell growth inhibition pathway, and that it might be a tumor suppressor in MM cells.

\section{Discussion}

Traditional Chinese medicine is an inexpensive treatment strategy. However, for most treatments neither the underlying therapeutic mechanisms nor the molecular targets of active compounds are well defined. Here, we describe a mechanism in MM cells by which BB inhibits cell proliferation and induces apoptosis and cell cycle arrest (Figure 3) via the regulation of miR-21 (Figure 2A).

miR-21 is over-expressed in the vast majority of cancer types analyzed so far and is thus recognized as an important oncomir $[27,28]$. Inhibition of miR-21 suppressed cell

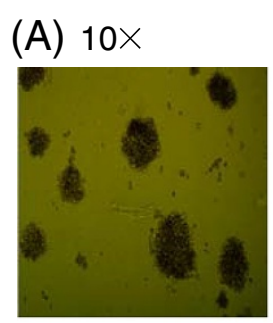

Blank

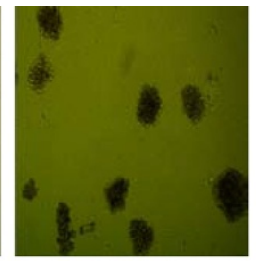

SCR



AMO-miR-21

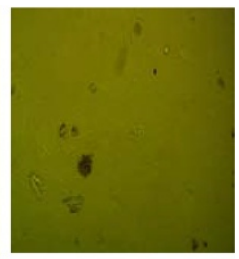

BB

(B)



Figure 4 BB and AMO-miR-21 suppress colony formation. Colony growth capacity was assessed by methylcellulose colony formation assays. (A) Images of representative colony formation. (B) After incubation for 1 week, RPMI-8266 cells treated with $75 \mu \mathrm{M}$ BB or $0.5 \mu \mathrm{M}$ AMO-miR-21 were observed under a light microscope and the number of colonies counted. Three independent experiments were carried out. 

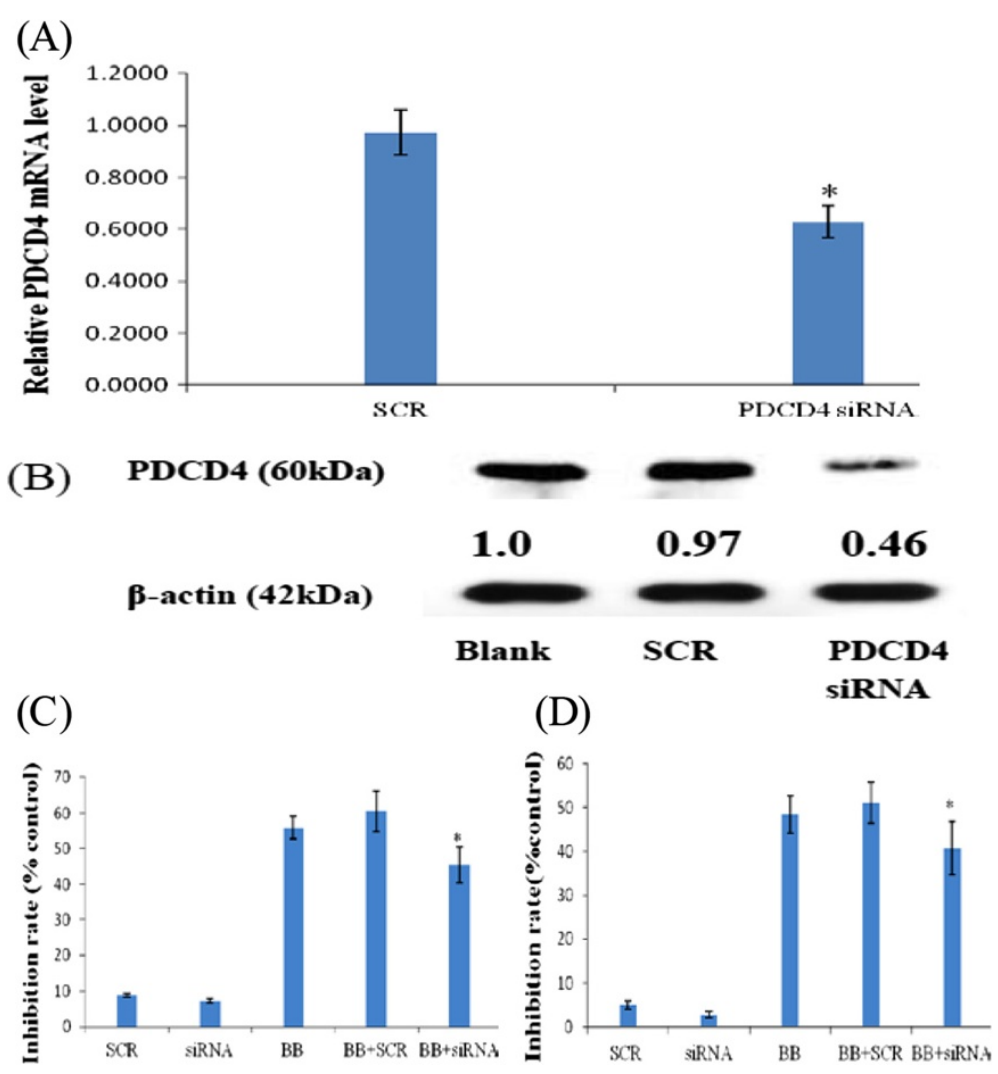

Figure 5 siRNA down-regulation of PDCD4 expression and rescue of BB-induced growth inhibition. RPMI-8266 cells were treated with 100 nM PDCD4 siRNA for $6 \mathrm{~h}$, and harvested $48 \mathrm{~h}$ after treatment. Total RNA and protein were isolated and analyzed for PDCD4 expression levels. (A) PDCD4 mRNA levels were determined by SYBR-Green real-time PCR. (B) PDCD4 protein levels were determined by western blot analysis. PDCD4 siRNA significantly down-regulated PDCD4 levels in RPMI-8266 cells. ${ }^{*} p<0.01$, vs. control. RPMI-8266 and U226 cells were pre-treated as above and then plated in 96-well plates in medium containing 10\% FCS for a further $72 \mathrm{~h}$ in the presence of BB (75 $\mu \mathrm{M})$. Cell viability was assessed by triplicate MTT assays. The results indicate that PDCD4 siRNA can rescue BB-induced growth inhibition. (C) RPMI-8266 cells. (D) U226 cells. ${ }^{*} p<0.01$ vs. control.

growth in culture and tumor growth in a xenograft mouse model [29]. Recently, Medina et al. demonstrated that miR21 over-expression leads to a pre-B malignant lymphoidlike phenotype and that inhibiting miR-21 alone induces complete tumor regression in a few days, suggesting that miR-21 is a central oncomiR in tumor formation [30]. Interestingly, $\mathrm{MM}$ is also a $\mathrm{B}$ cell neoplasm of clonal malignant cells in the bone marrow. Another group has also suggested that miR-21 as a key oncomiR in MM [29].

Our results show that $\mathrm{BB}$ down-regulates miR-21 levels in MM cell lines (RPMI-8266 and U226) (Figure 2A), but does not affect miR-21 levels in IL6-independent human myeloid leukemia cells (HL60, NB4, and K562 cell lines; data not shown).

Bioinformatic analysis identified two putative STAT3 binding sites upstream of the miR-21 gene (Additional file 1: Figure S4A), and STAT3 mRNA levels were significantly decreased by BB (Additional file 1: Figure S4B). STAT3 has been validated to be recruited to the miR-21 regulatory region in response to IL6 [16]. Our data indicate that IL6 levels in cell supernatants were significantly reduced by BB. Exogenous IL6 could partly ameliorate BB-mediated miR21 down-regulation (Figure 2A). Thus, in MM, downregulation of IL6 by BB might lead to the inhibition of miR-21 transcription through STAT3 down-regulation.

Antisense oligonucleotides targeting mRNAs have been successfully used to identify miRNA functions and for the development of therapeutic agents [31-33]. As miRNAs are small nucleic acids (19-24 nt), antisense inhibition is considered to be the best and possibly the only practical approach for specific pharmacological inhibition of their function [31]. Recently, an 8-mer nucleic acid complementary to the seed region of an miRNA, called a tiny antimiRNA, was used to antagonize miRNA function [25,26]. In this study, high levels of AMO-mir-21-FITC were detected by confocal microscopy, mainly in the cytoplasm (Additional file 1: Figure S6A), and $95.27 \%$ of cells were FITC positive at $24 \mathrm{~h}$ post-transfection (Additional file 1: Figure S6B). Here, we have investigated the anti-MM effects of AMO-miR-21 and compared them with those of BB. 
We also verified that $P D C D 4$ is a direct target of miR-21 (Additional file 1: Figure S5). Like AMO-miR-21, BB treatment down-regulated miR-21 and up-regulated PDCD4. Intriguingly, $\mathrm{BB}$ and $\mathrm{AMO}-\mathrm{miR}-21$ produced almost identical effects on cell proliferation, apoptosis, and cell cycle profile (Figure 3). Therefore, we conclude that the anti$\mathrm{MM}$ activity of $\mathrm{BB}$ might be achieved, at least in part, through the inhibition of miR-21/PDCD4 signaling.

The identification of miRNA target genes is necessary to assess the roles of aberrantly expressed miRNAs in human cancer and to subsequently develop miRNA-based gene therapies. miR-21 is strongly up-regulated in a variety of human neoplastic disorders. It can target and, therefore, can potentially regulate a number of important tumor suppressor genes (e.g., PDCD4, PTEN, TPM1, SPRY, RECK, and NFIB), which has attracted the attention of researchers in various fields [34,35]. PDCD4, a $64 \mathrm{kDa}$ protein, is an important, recently identified tumor suppressor that inhibits cell transformation, translation and invasion. PDCD4 is down-regulated in several types of human cancer [36-38], and is an independent predictor of poor prognosis in renal cell carcinoma patients [39]. Consistent with this notion, we show here that both $\mathrm{BB}$ and AMO-miR-21 upregulate $P D C D 4$ by suppressing miR-21 (Additional file 1: Figure S5A-B, Figure 2C-D). Silencing PDCD4 with siRNA rescued BB-induced cytotoxicity (Figure 5). Furthermore, PDCD4 modulates the expression of other genes on two levels. PDCD4 affects transcription of certain genes by inhibiting the activity of specific transcription factors, such as c-Jun [40,41], Sp1 [42] and p53 [43]. In addition, PDCD4 is thought to act as a suppressor of translation. A substantial body of evidence also suggests that PDCD4 is associated with p53 mRNA and suppresses its translation, thereby maintaining a low level of p53 in unstressed cells. This suppression is abrogated due to low levels of PDCD4 after DNA damage [44]. These observations are consistent with our predictions from the bioinformatic analysis (Figure 1).

Mechanisms of drug-insensitivity or resistance are involved in the apoptotic capacity of cancer cells [45]. Almost all cytotoxic anti-tumor drugs used clinically exert their effects by inducing apoptosis [46]. It is known that tumors escape apoptotic signals by expressing anti-apoptotic proteins, such as Bcl-2 family proteins $[45,46]$. Our study indicated that, similar to the effect caused by AMO-miR-21 inhibition of miR-21, BB treatment significantly promoted apoptosis, demonstrating the potential of $\mathrm{BB}$ and of targeting miR-21 for the treatment of MM (Figure 3). Hu et al. also show that miR-21 mediates berberine-induced apoptosis in human multiple myeloma cells [47].

\section{Conclusions}

To the best of our knowledge, our study is the first to show that the traditional Chinese medicine, BB, modulates the expression profile of many miRNAs in MM cells by down-regulating oncomirs and/or up-regulating tumor suppressor miRNAs. Our data show that BB treatment of MM cells down-regulated miR-21, probably via inhibition of IL6/STAT3, and led to the up-regulation of PDCD4, which was likely to result in suppression of the p53 signaling pathway. Therefore, $\mathrm{BB}$ is a therapeutic candidate for the treatment of MM (Additional file 1: Figure S10). Our work also provides new insight into the mechanisms underlying the anti-cancer effect of a traditional Chinese herbal medicine.

\section{Additional files}

\begin{abstract}
Additional file 1: Figure S1. Flowchart of Pathway Analysis. Figure S2. Sequences of AMO-miR-21 oligonucleotides and seed sequences in miR-21. Figure S3. Heat maps illustrating unsupervised clustering of mRNAs that were differentially expressed between the treated group (T-calibrated) and the normal group (N-calibrated). Figure S4. BB down-regulation of STAT3 mRNA level in MM cells. Figure S5. miR-21 directly targets the PDCD4 3' UTR. Figure S6. Transfection efficiency and localization of AMO-mir-21 in RPMI-8266 cells. Figure S7. BB and AMO-miR-21 induction of apoptosis. Figure S8. BB and AMO-miR-21 induction of G2-phase cell cycle arrest. Figure S9. KEGG analysis of p53 signaling pathways. Figure S10. Proposed pathway of BB inhibition of miRNA-21 in MM cells.
\end{abstract}

Additional file 2: Table S1. Oncomirs among down-regulated miRNAs. Table S2. Tumor suppressor genes among up-regulated miRNAs.

\section{Competing interest}

The authors declare that they have no competing interest.

\section{Authors' contributions}

JF conceived and designed the experiments. $X \mathrm{~L}, J \mathrm{~J}, \mathrm{RZ}$ and MF performed the experiments. JF and $Y L$ analyzed the data. $X Z$ and JF contributed reagents, materials and analytical tools. JF, YL and XZ wrote the paper. All authors read and approved the final manuscript.

\section{Acknowledgments}

This work was supported by grants from the National Natural Science Foundation of China (81170496), the Fundamental Research Funds for the Central Universities (no. 21609406), the Science and Technology Plan Projects of Guangdong Province, and the Key Discipline Construction Foundation of Jinan University. We are grateful to Chipscreening Inc. (Shenzhen, Guangdong, China) for microarray services and to Land biology Inc. (Guangzhou, China) for providing luciferase reporter services.

\section{Author details}

${ }^{1}$ Department of Biochemistry and Molecular Biology, Medical College of Jinan University, Guangzhou 510632, China. ${ }^{2}$ Department of Clinical Medicine, Medical College of Jinan University, Guangzhou 510632, China.

Received: 18 February 2014 Accepted: 29 May 2014

Published: 7 July 2014

\section{References}

1. Raab MS, Podar K, Breitkreutz I, Richardson PG, Anderson KC: Multiple myeloma. Lancet 2009, 374(9686):324-339

2. Ge F, Bi LJ, Tao SC, Xu XD, Zhang ZP, Kitazato K, Zhang XE: Proteomic analysis of multiple myeloma: current status and future perspectives. Proteomics Clin Appl 2011, 5(1-2):30-37.

3. Fonseca R, Barlogie B, Bataille R, Bastard C, Bergsagel PL, Chesi M, Davies FE, Drach J, Greipp PR, Kirsch IR, KuehI WM, Hernandez JM, Minvielle S, Pilarski LM, Shaughnessy JD Jr, Stewart AK, Avet-Loiseau H: Genetics and cytogenetics of multiple myeloma: a workshop report. Cancer Res 2004, 64(4):1546-1558.

4. Calin GA, Croce CM: MicroRNAs and chromosomal abnormalities in cancer cells. Oncogene 2006, 25(46):6202-6210. 
5. Calin GA, Croce CM: MicroRNA-cancer connection: the beginning of a new tale. Cancer Res 2006, 66(15):7390-7394.

6. Lionetti M, Biasiolo M, Agnelli L, Todoerti K, Mosca L, Fabris S, Sales G, Deliliers GL, Bicciato S, Lombardi L, Bortoluzzi S, Neri A: Identification of microRNA expression patterns and definition of a microRNA/ mRNA regulatory network in distinct molecular groups of multiple myeloma. Blood 2009, 114(25):e20-26.

7. Bartel DP: MicroRNAs: Genomics, biogenesis, mechanism, and function. Cell 2004, 116:281-297.

8. He L, Hannon GJ: MicroRNAs: Small RNAs with a big role in gene regulation. Nature Rev Genet 2004, 5:522-531.

9. Yu Z, Jian Z, Shen SH, Purisima E, Wang E: Global analysis of microRNA target gene expression reveals that miRNA targets are lower expressed in mature mouse and Drosophila tissues than in the embryos. Nucleic Acids Res 2007, 35:152-164.

10. Cho WC: OncomiRs: the discovery and progress of microRNAs in cancers. Mol Cancer 2007, 6:60.

11. Esquela-Kerscher A, Slack FJ: Oncomirs-microRNAs with a role in cancer. Nat Rev Cancer 2006, 6:259-269.

12. Pichiorri F, De Luca L, Aqeilan RI: MicroRNAs: new players in multiple myeloma. Front Genet 2011, 2:22

13. Munker R, Liu CG, Taccioli C, Alder H, Heerema N: MicroRNA profiles of drug-resistant myeloma cell lines. Acta Haematol 2010, 123(4):201-204.

14. Pichiorri F, Suh SS, Ladetto M, Kuehl M, Palumbo T, Drandi D, Taccioli C, Zanesi N, Alder H, Hagan JP, Munker R, Volinia S, Boccadoro M, Garzon R, Palumbo A, Aqeilan Rl, Croce CM: MicroRNAs regulate critical genes associated with multiple myeloma pathogenesis. Proc Natl Acad Sci U S A 2008, 105(35):12885-12890.

15. Xiong Q, Zhong Q, Zhang J, Yang M, Li C, Zheng P, Bi LJ, Ge F: Identification of novel mir-21 target proteins in multiple myeloma cells by quantitative proteomics. J Proteome Res 2012, 11(4):2078-2090.

16. Löffler D, Brocke-Heidrich K, Pfeifer G, Stocsits C, Hackermüller J, Kretzschmar AK, Burger R, Gramatzki M, Blumert C, Bauer K, Cvijic H, Ullmann AK, Stadler PF, Horn F: Interleukin- 6 dependent survival of multiple myeloma cells involves the Stat3-mediated induction of microRNA-21 through a highly conserved enhancer. Blood 2007, 110(4):1330-1333.

17. Zhang X, Gu L, Li J, Shah N, He J, Yang L, Hu Q, Zhou M: Degradation of MDM2 by the interaction between berberine and DAXX leads to potent apoptosis in MDM2-overexpressing cancer cells. Cancer Res 2010, 70(23):9895-9904

18. Pandey MK, Sung B, Kunnumakkara AB, Sethi G, Chaturvedi MM, Aggarwal BB: Berberine modifies cysteine 179 of IKBA kinase, suppresses nuclear factor-KB-regulated antiapoptotic gene products, and potentiates apoptosis. Cancer Res 2008, 68(13):5370-5379.

19. Yu FS, Yang JS, Lin HJ, Yu CS, Tan TW, Lin YT, Lin CC, Lu HF, Chung JG: Berberine inhibits WEHI-3 leukemia cells in vivo. In Vivo 2007, 21:407-412.

20. Mantena SK, Sharma SD, Katiyar SK: Berberine inhibits growth, induces G1 arrest and apoptosis in human epidermoid carcinoma A431 cells by regulating Cdki-Cdk-cyclin cascade, disruption of mitochondrial membrane potential and cleavage of caspase 3 and PARP. Carcinogenesis 2006, 27:2018-2027.

21. Anis KV, Rajeshkumar NV, Kuttan R: Inhibition of chemical carcinogenesis by berberine in rats and mice. J Pharm Pharmacol 2001, 53:763-768.

22. Nishino $H$, Kitagawa $K$, Fujiki $H$, Iwashima A: Berberine sulfate inhibits tumor-promoting activity of teleocidin in two-stage carcinogenesis on mouse skin. Oncology 1986, 43:131-134.

23. Sun $Y$, Xun $K$, Wang $Y$, Chen $X$ : A systematic review of the anticancer properties of berberine, a natural product from Chinese herbs. Anticancer Drugs 2009, 20:757-769.

24. Wang $X$ : Composition of seed sequence is a major determinant of microRNA targeting patterns. Bioinformatics 2014, 2014 Feb 12. [Epub ahead of print].

25. Rottiers V, Obad S, Petri A, McGarrah R, Lindholm MW, Black JC, Sinha S, Goody RJ, Lawrence MS, deLemos AS, Hansen HF, Whittaker S, Henry S, Brookes R, Najafi-Shoushtari SH, Chung RT, Whetstine JR, Gerszten RE, Kauppinen S, Näär AM: Pharmacological inhibition of a microRNA family in nonhuman primates by a seed-targeting 8-mer antimiR. Sci Transl Med 2013, 5(212):212ra162.

26. Obad S, dos Santos CO, Petri A, Heidenblad M, Broom O, Ruse C, Fu C, Lindow M, Stenvang J, Straarup EM, Hansen HF, Koch T, Pappin D, Hannon
GJ, Kauppinen S: Silencing of microRNA families by seed-targeting tiny LNAs. Nat Genet 2011, 43(4):371-378.

27. Lu Z, Liu M, Stribinskis V: MicroRNA-21 promotes cell transformation by targeting the programmed cell death 4 gene. Oncogene 2008, 27:4373-4379.

28. Asangani IA, Rasheed SA, Nikolova DA, Leupold JH, Colburn NH, Post S, Allgayer H: MicroRNA-21 (miR-21) post-transcriptionally downregulates tumor suppressor Pdcd4 and stimulates invasion, intravasation and metastasis in colorectal cancer. Oncogene 2008 27:2128-2136.

29. Si ML, Zhu S, Wu H, Lu Z, Wu F, Mo YY: miR-21-mediated tumor growth. Oncogene 2007, 26(19):2799-2803.

30. Medina PP, Nolde M, Slack FJ: OncomiR addiction in an vivo model of microRNA-21-induced pre-B-cell lymphoma. Nature 2010, 467(7311):86-90.

31. Esau CC: Inhibition of microRNA with antisense oligonucleotides. Methods 2008, 44:55-60.

32. Cheng AM, Byrom MW, Shelton J, Ford LP: Antisense inhibition of human miRNAs and indications for an involvement of miRNA in cell growth and apoptosis. Nucleic Acids Res 2005, 33:1290-1297.

33. Weiler J, Hunziker J, Hall J: Anti-miRNA oligonucleotides (AMOs): ammunition to target miRNAs implicated in human disease? Gene Ther 2005, 13:496-502.

34. Krichevsky AM, Gabriely G: miR-21: a small multi-faceted RNA. J Cell Mol Med 2009, 13(1):39-53.

35. Selcuklu SD, Donoghue MT, Spillane C: miR-21 as a key regulator of oncogenic processes. Biochem Soc Trans 2009, 37(Pt 4):918-925.

36. Lankat-Buttgereit B, Göke R: The tumour suppressor Pdcd4: recent advances in the elucidation of function and regulation. Biol Cell 2009, 101(6):309-317.

37. Sonenberg N, Pause A: Protein synthesis and oncogenesis meet again. Science 2006, 314(5798):428-429.

38. Leupold JH, Asangani IA, Mudduluru G, Allgayer H: Promoter cloning and characterization of the human programmed cell death protein 4 (pdcd4) gene: evidence for ZBP-89 and Sp-binding motifs as essential Pdcd4-regulators. Biosci Rep 2012, 32(3):281-297.

39. Li X, Xin S, Yang D, Li X, He Z, Che X, Wang J, Chen F, Wang X, Song X: Down-regulation of PDCD4 expression is an independent predictor of poor prognosis in human renal cell carcinoma patients. J Cancer Res Clin Oncol 2012, 138(3):529-535.

40. Yang HS, Knies JL, Stark C, Colburn NH: Pdcd4 suppresses tumor phenotype in JB6 cells by inhibiting AP-1 transactivation. Oncogene 2003, 22:3712-3720.

41. Bitomsky N, Böhm M, Klempnauer KH: Transformation suppressor protein Pdcd4 interferes with JNK-mediated phosphorylation of c-Jun and recruitment of the coactivator $\mathrm{p} 300$ by c-Jun. Oncogene 2004, 23(45):7484-7493

42. Leupold JH, Yang HS, Colburn NH, Asangani I, Post S, Allgayer H: Tumor suppressor Pdcd4 inhibits invasion/intravasation and regulates urokinase receptor (u-PAR) gene expression via Sp-transcription factors. Oncogene 2007, 26(31):4550-4562.

43. Bitomsky N, Wethkamp N, Marikkannu R, Klempnauer KH: siRNA-mediated knockdown of Pdcd4 expression causes upregulation of p21(Waf1/Cip1) expression. Oncogene 2008, 27(35):4820-9. Epub 2008 Apr 21.

44. Wedeken L, Singh P, Klempnauer KH: Tumor suppressor protein Pdcd4 inhibits translation of p53 mRNA. J Biol Chem 2011, 286(50):42855-62. Epub 2011 Oct 27.

45. Fraser M, Leung BM, Yan X, Dan HC, Cheng JQ, Tsang BK: p53 is a determinant of X-linked inhibitor of apoptosis protein/Akt-mediated chemoresistance in human ovarian cancer cells. Cancer Res 2003, 63:7081-7088.

46. Fraser M, Leung B, Jahani-Asl A, Yan X, Thompson WE, Tsang BK: Chemoresistance in human ovarian cancer: the role of apoptotic regulators. Reprod Biol Endocrinol 2003, 1:66.

47. Hu HY, Li KP, Wang XJ, Liu Y, Lu ZG, Dong RH, Guo HB, Zhang MX: Set9, NF-KB, and microRNA-21 mediate berberine-induced apoptosis of human multiple myeloma cells. 2013, 34(1):157-166.

doi:10.1186/1752-0509-8-82

Cite this article as: Luo et al:: Integrative analysis of differential miRNA and functional study of miR-21 by seed-targeting inhibition in multiple myeloma cells in response to berberine. BMC Systems Biology 2014 8:82. 